\title{
Swahili Poetry
}

THE East African Swahili Committee has published, as a supplement to No. 25 (June 1955) of its Journal, a long Swahili poem describing the German conquest of the Swahili coast. The poem, which is entitled Utenzi wa vita vya wadachi Kutamalaki Mrimi, consists of $63 \mathrm{I}$ stanzas and was written by Hemedi bin Abdallah bin Said el Buhriy in I 89I, five years after the events it describes. Mr. J. W. T. Allen, who has provided an English prose translation together with an Introduction and Notes, points out that this poem, unlike other Swahili tenzi, was composed and written by Hemedi bin Abdallah and is not based on an earlier version. He adds: 'Historically it is a remarkable work . . . a plain record of fact and genuine autobiography ... the feelings of the conquered people are vivid and convincing.'

While the original rhyming metre is preserved, the Arabic script is presented in roman type and in the standard Swahili orthography, the aim being to produce a text which can be easily read by anyone literate in Swahili. The translator has allowed himself a certain liberty in omitting repetitions.

In a Foreword Mr. W. Whiteley, Secretary of the East African Swahili Committee, referring to the fact that Swahili poetry is not widely known and not readily available to the general reader, expresses the hope that this publication will arouse interest and encourage the Committee to produce other examples of this literature.

\section{Le frangais à l'école primaire en Afrique}

Dans les écoles du Vicariat de Bukavu (Congo Belge) on enseigne désormais la lecture en français dès le début de l'école primaire. Dans le Bulletin trimestriel du C.E.P.S.I. (décembre, 1955) le R. P. Defour donne des précisions sur cette expérience. Au passé on a enseigné en kiswahili, mais ce n'est pas la langue maternelle des élèves; de plus, cette langue n'est pas aimée par la population des régions bashi et banyaruanda et les enfants, surtout les filles, s'obstinent parfois à ne pas la parler au début de leur écolage. Selon le système adopté maintenant, dès la première année le calcul, la lecture et le système métrique se donnent en français, le reste en kiswahili ou en dialecte local; en deuxième année on introduit deux heures par semaine de lecture en dialecte local; en quatrième année on commence à donner en français certaines explications, certaines causeries; en cinquième, tous les cours se donnent en français, sauf la grammaire et la lecture swahili. L'auteur trouve que l'enseignement du français est nécessaire non seulement aux élèves qui entreprendront des études secondaires, mais aussi à ceux qui ne continuent pas leurs études; ils en ont besoin pour leurs rapports avec les Européens. De plus les enfants doivent être capables de passer dans une école pour Européens. Il trouve, aussi, que les parents considèrent qu'il faut enseigner leurs enfants en français, que les élèves étudient mieux et avec plus d'ardeur et que les moniteurs désirent enseigner en français. En conclusion le Révérend Père, se référant à la communauté belgocongolaise, dit : ' Cette communauté doit être ouverte à tous et non seulement aux quelques diplômés de l'enseignement secondaire. ... Cette communauté ne se fera que si l'on se comprend; ce n'est pas ce baragouin de kiswahili à l'européenne qui permettra l'échange d'idées, le contact humain.'

\section{Institut Français d'Afrique Noire, Centre de Côte d'Ivoire}

Au cours des années 1955-1956, les activités de la Section d'Ethnologie-Sociologie, dirigée par B. Holas, intéressaient en premier lieu l'observation continue des sociétés éburnéennes en changement. Plusieurs missions de durée variable ont été organisées dans les différentes régions de la Côte d'Ivoire, et en partie au Soudan Français, en Haute-Volta, en Guinée Française.

Les principaux sujets étudiés étaient les suivants: $\mathbf{1}$. L'évolution actuelle du complexe 